\title{
Descrição do crescimento de ovinos Santa Inês utilizando modelos não-lineares selecionados por análise multivariada
}

\author{
Description of Santa Ines sheep growth using non-linear models selected by \\ multivariate analysis
}

\section{TEIXEIRA NETO, Milton Rezende ${ }^{{ }^{*}}$; CRUZ, Jurandir Ferreira da ${ }^{2}$; FARIA, Helder Henrique Neves ${ }^{3}$; SOUZA, Emanuely Santos ${ }^{3}$; CARNEIRO, Paulo Luiz Souza ${ }^{4}$; MALHADO, Carlos Henrique Mendes ${ }^{4}$}

\footnotetext{
${ }^{1}$ Universidade Estadual do Sudoeste da Bahia, Programa de Pós-Graduação em Zootecnia, Itapetinga, Bahia, Brasil.

${ }^{2}$ Universidade Estadual do Sudoeste da Bahia, Departamento de Fitotecnia e Zootecnia, Vitória da Conquista, Bahia, Brasil.

${ }^{3}$ Universidade Estadual do Sudoeste da Bahia, Vitória da Conquista, Bahia, Brasil.

${ }^{4}$ Universidade Estadual do Sudoeste da Bahia, Departamento de Ciências Biológicas, Jequié, Bahia, Brasil.

*Endereço para correspondência: rezendeteixeira@yahoo.com.br
}

\section{RESUMO}

O objetivo deste estudo foi descrever o crescimento de ovinos Santa Inês utilizando modelos não-lineares selecionados por análise multivariada. Dezessete modelos não-lineares foram utilizados para descrever o crescimento de cordeiros do nascimento aos 210 dias de idade. Os modelos foram classificados por meio de análise multivariada utilizando o coeficiente de determinação ajustado, critério de informação de Akaike, critério de informação Bayesiano, erro quadrático médio de predição, coeficiente de determinação de predição, desvio médio absoluto dos resíduos e percentual de convergência como avaliadores da qualidade do ajuste. Os modelos Richards, Reynolds e Weibull não convergiram e os modelos Schnute, Meloun II, IV e VI, Gamito, Michaelis Menten e Michaelis Menten Modificado não apresentaram padrão de crescimento tradicional. Os modelos Mitscherlich, Gompertz, Logístico, Meloun I, III e V, Brody e Von Bertalanffy convergiram e foram utilizados na análise de agrupamento. Dentre os quatro clusters formados, o primeiro, composto pelos modelos Mitscherlich, Meloun I e Brody, apresentou os melhores valores para os critério de informação de Akaike e bayesiano. Os parâmetros da curva foram influenciados pelo sexo e o tipo de parto $(\mathrm{P}<0,05)$ e o ponto inflexão ocorreu aos 94 dias de idade. Os modelos Mitscherlich, Meloun I e
Brody são adequados e equivalentes para descrição do crescimento de ovinos Santa Inês.

Palavras-chave: análise de agrupamento, cordeiro, curva de crescimento, ponto de inflexão, taxa de crescimento

\section{SUMMARY}

The objective of this study was to describe the growth of Santa Ines sheep using nonlinear models selected by multivariate analysis. Seventeen nonlinear models were used to describe the growth of sheep from birth to 210 days of age. The models were classified by means of multivariate analysis using the adjusted coefficient of determination, the Akaike information criterion, the Bayesian information criterion, the mean squared error of prediction, the coefficient of determination, mean absolute deviation and percentage of convergence of prediction as evaluators of adjustment quality. The Richards, Reynolds and Weibull models did not converge and the Schnute, Meloun II, IV and VI, Gamito, Michaelis Menten and Modified Michaelis Menten models did not show any traditional growth pattern. Models Mitscherlich, Gompertz, Logistic, Meloun I, III and V, Brody and Von Bertalanffy converged and were used in grouping analysis. Of the four formed clusters 
formed, the first, composed by models Mitscherlich, Meloun I and Brody, showed the best values for all the evaluators considered. The curve parameters were influenced by gender and type of birth $(\mathrm{P}<0.05)$ and the inflection point occurred at 94 days of age. The Mitscherlich, Meloun I and Brody models are adequate and equivalent to describe the growth of Santa Ines sheep.

Keywords: grouping analysis, growth curve, growth rate, lamb, point of inflection

\section{INTRODUÇÃO}

Diferentes modelos não-lineares têm sido utilizados para descrever o crescimento de ovinos, sendo os modelos de Richards, Gompertz, Brody, Logístico e Von Bertalanffy, os mais comuns (LÔBO et al., 2006; MALHADO et al., 2009; CHALH \& EL GAZZAH, 2014). Alternativamente, os modelos Schnute, Mitscherlich, Gamito e Meloun I, II, III e IV têm sido propostos (SILVEIRA et al., 2011).

Os modelos Schnute, Mitscherlich e Meloun I e II, utilizados anteriormente para descrever o crescimento da estrutura auricular de fetos (MELOUN \& MILITKY, 1996), devido à grande flexibilidade podem ser adequados para descrição de curvas de crescimento de animais.

Na maioria dos estudos sobre curvas de crescimento animal o coeficiente de determinação ajustado tem sido utilizado como critério de seleção do modelo mais adequado. No entanto o critério de informação de Akaike, critério de informação Bayesiano, erro quadrático médio de predição e o coeficiente de determinação de predição são avaliadores importantes da qualidade do ajuste do modelo que podem ser considerados (GÓMEZ et al., 2008).
Quanto maior o número de critérios considerados, mais segura é a indicação dos melhores modelos. Por outro lado, quando o número de avaliadores é grande, a escolha dos modelos pode se constituir em um processo complexo (SILVEIRA et al., 2011). A utilização de técnicas de agrupamento com base nas médias de cada avaliador permite a identificação dos melhores modelos de forma mais fácil (SILVEIRA et al., 2012).

Este estudo teve como objetivo identificar, por meio de análise multivariada e avaliadores de qualidade, o modelo não-linear mais adequado para descrever o crescimento de ovinos da raça Santa Inês.

\section{MATERIAL E MÉTODOS}

Os dados foram coletados no período de setembro de 2011 a maio de 2012, na Fazenda União, localizada no município de Ribeirão do Largo-BA (15'27'33" S e $\left.40^{\circ} 44^{\prime} 28^{\prime \prime}\right)$. Foram utilizados 89 ovinos puros da raça Santa Inês, sendo 43 machos e 46 fêmeas. Os animais foram criados em sistema semi-intensivo e mantidos com as mães até a idade de 90 dias, momento em que ocorreu o desmame.

A alimentação das mães foi constituída de pastagem de grama estrela e as crias receberam suplementação com alimento concentrado (16\% PB) em quantidade equivalente a $1,0 \%$ peso vivo/dia.

A pesagem dos animais foi realizada sistematicamente em intervalos de 30 dias, desde o nascimento até os 210 dias de idade. O controle parasitário foi realizado com base em exames parasitológicos de fezes (OPG) conforme programa profilático da propriedade. 
Para determinação dos parâmetros da curva de crescimento foram utilizados os modelos não-lineares de Richards, Brody, Gompertz, Logístico, Von Bertalanffy, Reynolds, Weibull, Mitscherlich, Schnute, Gamito, Michaelis Menten, Michaelis Menten Modificado e Meloun I, II, III, IV e V (Tabela 1). Para todos os modelos utilizados, o parâmetro $\beta_{1}$ representou o peso adulto, ou peso assintótico, do animal; o parâmetro $\beta_{3}$, a taxa de maturidade, ou velocidade de crescimento; o parâmetro $\beta_{4}$, o ponto de inflexão; e o parâmetro $\beta_{2}$, uma constante de integração.

Tabela 1. Modelos de regressão não-linear utilizados para descrição da curva de crescimento dos cordeiros

\begin{tabular}{|c|c|c|c|}
\hline Modelo & Equação & Modelo & Equação \\
\hline A Schnute & $y_{i}=\frac{\beta_{1}}{\left(1+\beta_{4} e^{\left(\beta_{3} \beta_{2}-x_{i}\right)}\right) \frac{1}{\beta_{4}}}+e_{i}$ & J Reynolds & $y_{i}=\beta_{1}+\left(\beta_{2} x_{i}\right)^{\frac{-1}{\beta_{2}}}+e_{i}$ \\
\hline B Mitscherlich & $y_{i}=\beta_{1}\left(1-e^{\left(\beta_{3} \beta_{2}-\beta_{3} x_{i}\right)}\right)+e_{i}$ & K Gamito & $y_{i}=\frac{1}{\left(\beta_{1}+\beta_{2} x_{i} \beta_{3}\right)}+e_{i}$ \\
\hline C Richards & $y_{i}=\frac{\beta_{1}}{\left(1+e^{\left(\beta_{2}-\beta_{3} x_{i}\right)}\right) \frac{1}{\beta_{4}}}+e_{i}$ & L Meloun V & $y_{i}=\frac{\beta_{2} \beta_{3}+\beta_{1} \beta_{4} x_{i}}{\beta_{3}+x_{i} \beta_{4}}+e_{i}$ \\
\hline D Gompertz & $y_{i}=\beta_{1} e^{\left(-e^{\left(\beta_{2}-\beta_{3} x_{i}\right)}\right)}+e_{i}$ & M Meloun VI & $y_{i}=\beta_{1}-\beta_{2} e^{\left(-\beta_{3} x_{i} \beta_{4}\right)}+e$ \\
\hline E Logístico & $y_{i}=\frac{\beta_{1}}{\left(1+e^{\left(\beta_{2}-\beta_{3} x_{i}\right)}\right)}+e_{i}$ & N Brody & $y_{i}=\beta_{1}\left(1-\beta_{2} e^{-\beta_{3} x_{i}}\right)+e_{i}$ \\
\hline F Meloun I & $y_{i}=\beta_{1}-\beta_{2} e^{\left(-\beta_{3} x_{i}\right)}+e_{i}$ & O von Bertalanffy & $y_{i}=\beta_{1}\left(1-\beta_{2} e^{-\beta_{3} x_{i}}\right)^{3}+\epsilon$ \\
\hline G Meloun II & $y_{i}=\beta_{1}\left(1-e^{\left(-x-\beta_{2}\right)} \beta_{3}\right)+e_{i}$ & P Michaelis-Meten & $y_{i}=\frac{\beta_{1} x_{i}}{x_{i}+\beta_{2}}+e_{i}$ \\
\hline H Meloun III & $y_{i}=\beta_{1}-e^{\left(-\beta_{2}-\beta_{3} x_{i}\right)}+e_{i}$ & $\begin{array}{l}\text { Q Michaelis-Meten } \\
\text { Modified }\end{array}$ & $y_{i}=\frac{\beta_{2} \beta_{3}^{\beta_{4}}+\beta_{1} x_{i}^{\beta_{4}}}{\beta_{3}^{\beta_{4}}+x_{i}^{\beta_{4}}}+e_{i}$ \\
\hline I Meloun IV & $y_{i}=\beta_{1}-\beta_{2} e^{\left(-\beta_{3} x \beta_{4}\right)}+e_{i}$ & R Weibull & $y_{i}=\beta_{1}-\beta_{2} e^{-e^{\beta_{3}} x_{i}^{\beta_{4}}}+e_{i}$ \\
\hline
\end{tabular}

Todos os modelos foram ajustados pelo método da Máxima Verossimilhança via algoritmo de otimização de GaussNewton utilizando o PROC MODEL ${ }^{\circledR}$ versão 9.1 (SAS, 2003). Os valores dos parâmetros foram definidos após identificação daqueles que apresentavam menor número de interações para convergência.
Para avaliar a qualidade do ajuste dos modelos foram utilizados como avaliadores o coeficiente de determinação ajustado $\left(\mathrm{R}_{\text {aj }}^{2}\right)$, critério de informação de Akaike (AIC), critério de informação bayesiano (BIC), erro quadrático médio de predição (MEP), coeficiente de determinação de predição $\left(\mathrm{R}_{\mathrm{p}}^{2}\right)$, percentual de convergência $(\mathrm{C} \%)$ e 
desvio médio absoluto dos resíduos (DMA), conforme sugerido por Silveira et al. (2011) e Sarmento et al. (2006).

Após constatação da convergência, os valores dos avaliadores de cada modelo foram calculados a partir da média dos valores dos avaliadores obtidos para cada animal. Posteriormente, os dados foram submetidos à análise de agrupamento (PROC CLUSTER, SAS, versão 9.1).

Depois de selecionado o modelo, foi avaliada a influência do sexo e tipo de parto sobre os parâmetros da curva (GLM, SAS, versão 9.1), bem como foram estimadas as correlações de Pearson entre os parâmetros e a taxa de crescimento absoluto (TCA). Para os modelos que não apresentam ponto de inflexão utilizou-se o método da máxima curvatura geométrica para determinar a idade em que os animais desaceleram o ganho de peso, conforme sugerido por Cecon et al. (2008).

\section{RESULTADOS E DISCUSSÃO}

Os modelos Richards, Reynolds e Weibull não convergiram e os modelos Schnute, Meloun II, IV e VI, Gamito, Michaelis Menten e Michaelis Menten Modificado não apresentaram padrão de crescimento tradicional e, por essa razão seus parâmetros não foram considerados. Os modelos Mitscherlich, Gompertz, Logístico, Meloun I, III e V, Brody e Von Bertalanffy mostraram-se adequados, sendo o Meloun $\mathrm{V}$ o único modelo com quatro parâmetros (Tabela 2).

Tabela 2. Valores de peso assintótico $\left(\beta_{1}\right)$, constante de integração $\left(\beta_{2}\right)$, taxa de maturidade $\left(\beta_{3}\right)$ e ponto de inflexão $\left(\beta_{4}\right)$, estimados por diferentes modelos de crescimento

\begin{tabular}{lcrcc}
\hline \multirow{2}{*}{ Modelo } & \multicolumn{4}{c}{ Parâmetros } \\
\cline { 2 - 5 } & $\beta_{1}$ & \multicolumn{1}{c}{$\beta_{2}$} & $\beta_{3}$ & $\beta_{4}$ \\
\hline B - Mitscherlich & 48,8903 & $-14,1364$ & 0,0063 & - \\
D - Gompertz & 37,5630 & 0,7200 & 0,0147 & - \\
E - Logístico & 34,7452 & 1,6076 & 0,0229 & - \\
F - Meloun I & 48,8897 & 45,3617 & 0,0063 & - \\
H - Meloun III & 48,8884 & $-3,7805$ & 0,0063 & - \\
L - Meloun V & 66,5421 & 3,3602 & 70,3911 & 0,3000 \\
N - Brody & 48,8893 & 0,9242 & 0,0063 & - \\
O - Von Bertalanffy & 39,6772 & 0,5194 & 0,0119 & - \\
\hline
\end{tabular}

Com exceção do modelo Meloun V, todos os demais modelos superestimaram o peso ao nascimento. O peso assintótico estimado $\left(\beta_{1}\right)$ variou de 37,56 a $66,54 \mathrm{~kg}$, sendo que os modelos Mitscherlich, Meloun I e III e Brody apresentaram estimativas semelhantes.

O peso assintótico representa à estimativa do peso à maturidade e não o peso máximo que o animal pode alcançar
(BROW et al., 1976) e a variação verificada na sua estimativa nos diferentes modelos pode ser atribuída à dificuldade dos modelos não-lineares em ajustar o peso inicial, comprometendo o processo de estimação desse parâmetro, fato esse também verificado por Cruz et al. (2009). A estimativa do $\beta_{1}$ pode muitas vezes ser sujeita a erros porque o conjunto de dados ajustado por modelos 
não-lineares de crescimento não inclui o crescimento do animal até a maturidade (TORAL, 2008).

Por outro lado, a estimativa do peso assintótico para uma determinada raça pode variar em função de outros fatores. Guedes et al. (2004) utilizando os modelos Brody, Von Bertalanffy, Gompertz e Logístico para descrever o crescimento de animais Santa Inês criados em sistema intensivo encontraram valores superiores aos verificados no presente estudo $(65,28 ; 51,06 ; 43,18$ e $37,24 \mathrm{~kg}$, respectivamente). Nesse caso, a diferença verificada entre os dois estudos pode ser devida ao manejo dos animais, uma vez que estimativa do peso assintótico pode sofrer influencia da raça e do sistema de criação (MALHADO et al., 2008).

A taxa de maturidade $\left(\beta_{3}\right)$ apresentou grande amplitude, cujos valores variaram de 0,0063 a 70,3911. Considerando que a taxa de maturidade indica a velocidade de crescimento para atingir o peso assintótico, os valores elevados de $\beta_{3}$ podem ser interpretados como indicadores de precocidade.

Todos os modelos apresentaram bons ajustes em relação aos coeficientes de determinação ajustado $\left(\mathrm{R}_{\text {aj }}^{2}\right)$ e de predição $\left(\mathrm{R}_{\mathrm{p}}^{2}\right)$, com médias superiores a 0,97 . O critério de informação de Akaike (AIC) apresentou menores médias para Brody e Meloun I, enquanto que, para critério de informação Bayesiano (BIC) o menor valor foi verificado para o modelo Brody. O erro quadrático médio de predição (MEP) apresentou menor e maior média para os modelos Meloun I e Meloun V, respectivamente. Com base no percentual de convergência (C\%), os modelos Gompertz, Logístico e Von Bertalanffy foram superiores aos demais, convergindo a curva de todos os animais. No entanto, esses modelos apresentaram os maiores valores de desvio médio absoluto (Tabela 3 ).
Os modelos com melhor ajuste apresentam maiores valores de coeficiente de determinação $\left(\mathrm{R}_{\text {aj }}^{2}\right.$ e $\left.\mathrm{R}_{\mathrm{p}}^{2}\right)$ e percentual de convergência e menores AIC, BIC, MEP DMA (SILVA et al., 2011; SARMENTO et al., 2006). Em estudo com ovinos F1 Santa Inês $x$ Dorper Silveira et al., (2011) encontraram médias semelhantes as do presente estudo para $\mathrm{R}_{\text {aj }}^{2}(0,9914), \mathrm{R}_{\mathrm{p}}^{2}$ $(0,9992), \quad$ AIC $(25,9349)$ e BIC $(27,9102)$ e inferior para $\operatorname{MEP}(0,6708)$. Todos os avaliadores de qualidade, com exceção do MEP e DMA, apresentaram baixo coeficiente de variação $(\mathrm{CV})$. $\mathrm{O}$ alto $\mathrm{CV}$ obtido para o MEP deveu-se ao fato de este avaliador ser calculado após a remoção sucessiva de observações do conjunto de dados originais, avaliando a capacidade do modelo de se ajustar na ausência de informações (NOBRE et al., 1987). Quanto ao DMA, a dificuldade dos modelos para estimar o peso ao nascimento proporcionou diferentes estimativas do peso nas subsequentes idades, contribuindo para aumentar o CV.

A amplitude observada para o $\mathrm{C} \%$, parâmetro o qual expressa a quantidade de animais que o modelo conseguiu convergir a curva de crescimento, foi resultante do menor número de curvas traçadas pelo modelo com quatro parâmetros (Meloun V), evidenciando a dificuldade de se trabalhar com modelos mais parametrizados.

A importância de cada avaliador da qualidade de ajuste depende do $\mathrm{CV}$, sendo que baixos valores indicam equivalência dos modelos em relação ao avaliador. Considerando que as diferenças entre os modelos para os avaliadores $R_{a j}^{2}$ e $\quad R_{p}^{2}$ foram irrelevantes, o AIC e BIC contribuíram estrategicamente para definição do modelo com melhor ajuste. 
Rev. Bras. Saúde Prod. Anim., Salvador, v.17, n.1, p.26-36 jan./mar., 2016 http://www.rbspa.ufba.br ISSN 15199940

Tabela 3. Valores do critério de informação de Akaike (AIC), critério de informação Bayesiano (BIC), coeficiente de determinação ajustado $\left(\mathrm{R}^{2}{ }_{\mathrm{aj}}\right.$ ), erro quadrático médio de predição (MEP), coeficiente de determinação de predição $\left(\mathrm{R}_{\mathrm{p}}^{2}\right)$, percentual de convergência $(\mathrm{C} \%)$ e desvio médio absoluto dos resíduos (DMA) de acordo com os modelos estudados

\begin{tabular}{lccccccc}
\hline \multirow{2}{*}{ Modelo } & \multicolumn{7}{c}{ Avaliadores } \\
\cline { 2 - 8 } & $\mathrm{AIC}$ & $\mathrm{BIC}$ & $\mathrm{R}_{\mathrm{aj}}^{2}$ & $\mathrm{MEP}$ & $\mathrm{R}_{\mathrm{p}}^{2}$ & \%C & DMA \\
\hline B - Mitscherlich & 25,1300 & 25,3346 & 0,9820 & 3,9351 & 0,9962 & 93,26 & 1,4637 \\
D - Gompertz & 28,8647 & 29,1030 & 0,9705 & 5,5999 & 0,9941 & 100,00 & 1,9676 \\
E - Logístico & 28,9052 & 29,1436 & 0,9705 & 8,1912 & 0,9915 & 100,00 & 2,4169 \\
F - Meloun I & 25,1270 & 25,3316 & 0,9820 & 3,9334 & 0,9962 & 93,26 & 1,4635 \\
H - Meloun III & 28,5642 & 28,7584 & 0,9718 & 3,9364 & 0,9962 & 93,26 & 1,4638 \\
L - Meloun V & 28,5202 & 28,3776 & 0,9724 & 21,0371 & 0,9813 & 80,90 & 1,3197 \\
N - Brody & 25,1195 & 25,3144 & 0,9821 & 3,9345 & 0,9962 & 93,26 & 1,4636 \\
O - VonBertalanffy & 28,8451 & 29,0786 & 0,9705 & 4,6201 & 0,9951 & 100,00 & 1,7783 \\
\hline Média & 27,3845 & 27,5552 & 0,9752 & 6,8985 & 0,9934 & 94,24 & 1,6671 \\
Desvio-padrão & 1,8757 & 1,8616 & 0,0057 & 5,8989 & 0,0051 & 6,3396 & 0,3686 \\
CV (\%) & 6,8495 & 6,7561 & 0,5823 & 85,5108 & 0,5172 & 6,7269 & 22,1104 \\
\hline
\end{tabular}

Dentre os quatro clusters formados, o primeiro, composto pelos modelos Mitscherlich, Meloun I e Brody, apresentou as melhores médias para os avaliadores $\mathrm{AIC}, \mathrm{BIC}, \mathrm{R}_{\mathrm{aj}}^{2}, \mathrm{MEP}, \mathrm{R}_{\mathrm{p}}^{2} \mathrm{e}$
DMA. O segundo cluster, composto pelos modelos Meloun III, Von Bertalanffy e Gompertz, apresentou o maior valor para o percentual de convergência (Tabela 4).

Tabela 4. Clusters com respectivos modelos e médias dos avaliadores de qualidade do ajuste

\begin{tabular}{lcccc}
\hline & Cluster 1 & Cluster 2 & Cluster 3 & Cluster 4 \\
\cline { 2 - 4 } Avaliador & Modelos & Modelos & Modelo & Modelo \\
& $\mathrm{B}, \mathrm{F}, \mathrm{N}$ & $\mathrm{H}, \mathrm{O}, \mathrm{D}$ & $\mathrm{E}$ & $\mathrm{L}$ \\
\hline $\mathrm{AIC}$ & 25,1255 & 28,7580 & 28,9052 & 28,5202 \\
$\mathrm{BIC}$ & 25,3269 & 28,9800 & 29,1436 & 28,3776 \\
$\mathrm{R}_{\text {aj }}^{2}$ & 0,9820 & 0,9709 & 0,9705 & 0,9724 \\
MEP $^{2}$ & 3,9343 & 4,7188 & 8,1912 & 21,0371 \\
$\mathrm{R}_{\mathrm{p}}$ & 0,9962 & 0,9951 & 0,9915 & 0,9813 \\
DMA & 1,4636 & 1,7366 & 2,4169 & 1,3197 \\
$\mathrm{C} \%$ & 0,9326 & 0,9775 & 1,0000 & 0,8090 \\
\hline
\end{tabular}

$\mathrm{AIC}=$ critério de informação de Akaike; BIC $=$ critério de informação bayesiano; $\mathrm{R}^{2}{ }_{\mathrm{aj}}=$ coeficiente de determinação ajustado; MEP = erro quadrático médio de predição; $\mathrm{R}_{\mathrm{p}}^{2}=$ coeficiente de determinação de predição; DMA = desvio médio absoluto dos resíduos; $\mathrm{C} \%=$ percentual de convergência; $\mathrm{B}=$ Mitscherlich; D = Gompertz; E = Logístico; F = Meloun I; H = Meloun III; L = Meloun V; N = Brody; $\mathrm{O}=$ Von Bertalanffy. 
Apesar de alguns modelos não-lineares serem apontados como adequados para descrever o crescimento de ovinos Santa Inês, existe divergências quanto à definição do melhor modelo. Enquanto Lobo et al. (2006) recomendam o modelo Richards, Guedes et al. (2004) e Sarmento et al. (2006), indicam o modelo Gompertz, e Silva et al. (2012) o modelo logístico. Essa dificuldade de precisar o melhor modelo pode ser em função da desuniformidade dos estudos quanto a quantidade de dados, oscilação nos valores dos pesos, número de pesagens por animal e idade da última pesagem (TORAL, 2008).

No entanto, vale salientar que a dificuldade na definição do melhor modelo deve-se também às particularidades da população avaliada, uma vez que os ovinos Santa Inês, segundo Teixeira Neto et al. (2015), apresentam elevada variabilidade fenotípica.

$\mathrm{Na}$ escolha do modelo que melhor descreve o crescimento devem ser atendidos três pontos de igual importância: a interpretação biológica dos parâmetros, a qualidade de ajuste e o percentual de convergência (FITZHUGH JÚNIOR, 1976). Nas situações em que os modelos são similares em relação aos parâmetros e que o modelo com melhor ajuste não é o que apresenta maior percentual de convergência, a escolha do modelo mais adequado fica a critério do pesquisador, priorizando o que melhor descreve o crescimento ou o que retrata o crescimento do maior número de animais. De acordo com Cavalcante et al. (2013), o melhor modelo a ser utilizado é aquele que melhor se adapta e apresenta resultados mais adequados ao caso de estudo.

No presente estudo, ao priorizar a qualidade de ajuste, os modelos Mitscherlich, Meloun I e Brody mostraram-se equivalentes. No entanto, o modelo Brody apresentou valores ligeiramente melhores para AIC, BIC e $\mathrm{R}^{2}{ }_{\mathrm{aj}}$, sendo, por essa razão, escolhido para o cálculo das correlações e avaliação da influência do sexo e tipo de parto sobre os parâmetros da curva, bem como para descrever a taxa de crescimento absoluto (TCA) dos animais.

$\mathrm{O}$ efeito da interação sexo $\mathrm{x}$ tipo de parto não foi significativo $(\mathrm{P}>0,05)$. Animais oriundos de partos simples e duplos apresentaram parâmetros de crescimento diferentes $(\mathrm{P}<0,05)$, sendo que aqueles oriundos de partos simples apresentaram maior velocidade de crescimento. Por outro lado, os animais oriundos de parto duplo apresentaram maior peso à maturidade (Tabela 5).

Esse resultados corroboram com os achados por Sarmento et al. (2006), quando verificaram que animais com maiores taxas de crescimento têm menor probabilidade de atingir maiores pesos à maturidade que aqueles que crescem mais lentamente no início da vida.

Machos e fêmeas apresentaram taxa de crescimento semelhante $(\mathrm{P}>0,05)$. Entretanto, os machos apresentaram maior peso à maturidade $(\mathrm{P}<0,05)$. De forma diferente ao verificado no presente estudo, Souza et al. (2011) avaliando o crescimento de ovinos Morada Nova pelo modelo Von Bertalanffy verificaram que o sexo influenciou os parâmetros $\beta_{1}$ e $\beta_{3}$.

No presente estudo, verificou-se correlações negativas do parâmetro $\beta_{3}$ $\operatorname{com} \beta_{1}$ e $\beta_{2}(-0,76$ e $-0,50 ; \mathrm{P}<0,01)$. Por outro lado, os parâmetros $\beta_{1}$ e $\beta_{2}$ apresentaram correlação positiva elevada entre si $(0,75)$. Do Ó et al. (2012) e Lôbo et al. (2006) encontraram correlação negativa entre $\beta_{1}$ e $\beta_{3}$ de 0,26 e -0,87, em ovinos Santa Inês, respectivamente. 
Rev. Bras. Saúde Prod. Anim., Salvador, v.17, n.1, p.26-36 jan./mar., 2016 http://www.rbspa.ufba.br ISSN 15199940

Tabela 5. Peso assintótico $\left(\beta_{1}\right)$ constante de integração $\left(\beta_{2}\right)$ e taxa de maturidade $\left(\beta_{3}\right)$ estimados pelo modelo não-linear de Brody, de acordo com o sexo e tipo de parto para ovinos da raça Santa Inês

\begin{tabular}{lccc}
\hline \multirow{2}{*}{ Fonte de variação } & \multicolumn{3}{c}{ Média da estimativa dos parâmetros } \\
\cline { 2 - 4 } & $\beta_{1}$ & $\beta_{2}$ & $\beta_{3}$ \\
\hline Sexo & & & $0,0059237^{\mathrm{A}}$ \\
\hline Macho & $52,988^{\mathrm{A}}$ & $0,927874^{\mathrm{B}}$ & $0,0067287^{\mathrm{A}}$ \\
Fêmea & $45,076^{\mathrm{B}}$ & $0,920860^{\mathrm{B}}$ & $0,0079989^{\mathrm{A}}$ \\
\hline Tipo de parto & & $0,0045582^{\mathrm{B}}$ \\
\hline Simples & $43,680^{\mathrm{B}}$ & $0,915737^{\mathrm{B}}$ & \\
Duplo & $54,489^{\mathrm{A}}$ & $0,933382^{\mathrm{A}}$ & 0
\end{tabular}

Valores seguidos de mesma letra, na coluna, não diferiram pelo teste $\mathrm{t}(\mathrm{P}<0,05)$.

De acordo com Sarmento et al. (2006), uma possível conseqüência biológica da correlação negativa entre $\beta_{2}$ e $\beta_{3}$ seria que cordeiros desmamados mais leves (valores altos de $\beta_{2}$ ) tendem apresentar menores taxas de crescimento relativo (quociente entre ganho de peso e peso) $\mathrm{e}$, conseqüentemente, menores taxas de maturidade (menores valores de $\beta_{3}$ ), do que aqueles desmamados mais pesados. Por outro lado, a correlação positiva entre $\beta_{1}$ e $\beta_{2}$ poderia indicar que os cordeiros mais pesados ao desmame atingiriam maiores pesos à maturidade.

A relação biológica mais importante para uma curva está entre os parâmetros $\beta_{1}$ e $\beta_{3}$ (McMANUS et al., 2003). O interessante seria que animais de produção de carne apresentassem maior velocidade de crescimento e maturidade o mais precocemente possível, reduzindo o tempo que o animal necessitaria para atingir a idade reprodutiva, melhorando, assim, a taxa de retorno da atividade.

$\mathrm{O}$ ponto de inflexão da curva ocorreu aos 94 dias de idade, momento em que os animais apresentaram TCA de $0,158 \mathrm{~kg} /$ dia (Figura 1). A identificação do ponto de inflexão imediatamente após o desmame sugere a necessidade de ajuste no manejo nutricional para reduzir os efeitos da mudança de dieta sobre a TCA. Segundo Souza et al. (2013), esta informação é de importância estratégica para os produtores, auxiliando na determinação da idade mais econômica para abate, oferecendo a melhor relação custo / benefício.

As informações sobre o desempenho animal expressas pelo TCA torna possível a determinação da idade ideal de abate. Segundo Silva et al. (2011), após o ponto inflexão o consumo animal passa a ser destinado à mantença, ocorre redução da conversão alimentar e o custo para manutenção do animal é elevado. Desta forma o TCA possibilita a identificação do momento, em torno do qual o animal, com ganho de peso satisfatório, deve ser abatido ou descartado.

$\mathrm{O}$ ponto de inflexão e o TCA, evidentemente, podem ser influenciados por fatores ambientais e/ou genéticos. Estudos anteriores mostram ponto de inflexão e TCA de 200 dias de idade e $0,150 \mathrm{~kg} / \mathrm{dia}$ (SILVA et al., 2012) e de 52 dias de idade e $0,170 \mathrm{~kg} / \mathrm{dia}$ (SARMENTO et al., 2006), respectivamente. Além das diferenças ambientais, a variabilidade genética existente dentro da raça Santa Inês certamente contribui para ocorrência dessas variações. 

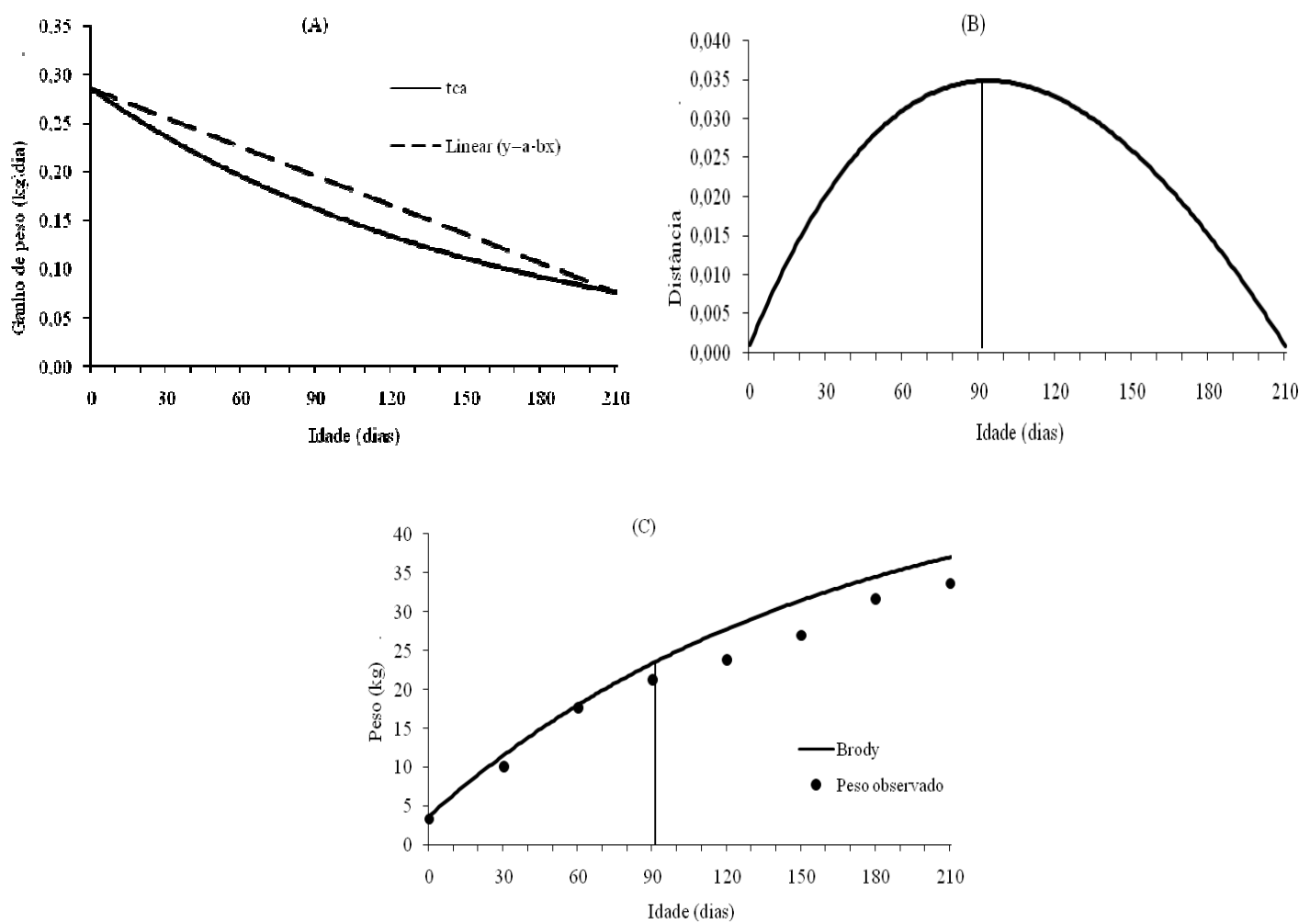

Figura 1. Taxa de crescimento absoluto, (A), ponto de máxima curvatura (B) e curva de crescimento estimada pelo modelo Brody (C) de ovinos Santa Inês

Assim, a análise multivariada dos avaliadores de qualidade de ajuste se constitui em um facilitador na identificação de modelos não lineares para descrição da curva de crescimento em ovinos Santa Inês. Os modelos Mitscherlich, Meloun I e Brody são adequados e equivalentes para descrição do crescimento, sendo que, o modelo Brody revela uma notável redução do ganho de peso após o desmame.

\section{REFERÊNCIAS}

BROW, J.E.; FITZHUGH JÚNIOR, H.A.; CARTWRIGHT, T.C.A. A comparison of nonlinear models for describing weigt-age relationships in cattle. Journal of Animal Science, v.42, p.810-818, 1976.
CAVALCANTE, D.H.; CAMPELO, J.E.G.; SOUSA JÚNIOR, S.C.; SOUSA, G.G.T.; ARAÚJO, J.I.M.; ARAÚJO, A.C.; FONSECA, W.J.L.; BARROS JÚNIOR, C.P.; ARAÚJO, A.M. Modelos não paramétricos para ajustes de curva de crescimento em caprinos Sem Raça Definida (SRD).

Revista Acadêmica: Ciências

Agrárias e Ambientais, v.11, n.3, p.283-289, 2013.

CECON, P.R.; SILVA, F.F.; FERREIRA, A.; FERRÃO, R.G.; CARNEIRO, A.P.S.; DETMANN, E.; FARIA, P.N.; MORAIS, T.S.S. Análise de medidas repetidas na avaliação de clones de café 'Conilon'. Pesquisa Agropecuária Brasileira, v.43, p.11711176, 2008. 
Rev. Bras. Saúde Prod. Anim., Salvador, v.17, n.1, p.26-36 jan./mar., 2016 http://www.rbspa.ufba.br ISSN 15199940

CHALH, A.; EL GAZZAH, M. Variogram investigation of covariance shape within longitudinal data with possible use of a krigeage technique as an interpolation tool: Sheep growth data as an example. Irish Journal of Agricultural and Food Research, v.53, p.51-64, 2014.

CRUZ, G.R.B.; COSTA, R.G.; RIBEIRO, M.N. Curva de crescimento de caprinos mestiços no estado da Paraíba. Revista Brasileira de Ciências Agrárias, v.4, n.2, p.204-210, 2009.

DO Ó, A.O.; RÊGO NETO, A.A.; SANTOS, G.V.; SARMENTO, J.L.R.; BIAGIOTTI, D.; SOUSA, J.E.R. Curva de crescimento de ovinos Santa Inês no Vale do Gurgueia. Revista Brasileira de Saúde e Produção Animal [online], v.13, n.4, p.912-922, 2012.

FITZHUGH JÚNIOR, H.A. Analysis of growth curves and strategies for altering their shape. Journal of Animal Science, v.42, n.4, p.1036-1051, 1976.

GÓMEZ, D.A.A.; MUÑOZ, M.F.C.; BETANCUR, L.F.R. Modelación de las funciones de crecimiento aplicadas a la producción animal. Revista

Colombiana de Ciencias Pecuárias, v.21, p.39-58, 2008.

GUEDES, M.H.P.; MUNIZ, J.A.; PEREZ, J.R.O.; SILVA, F.F.; AQUINO, L.H.; SANTOS, C.L. Estudo das curvas de crescimento de cordeiros das raças Santa Inês e Bergamácia considerando heterogeneidade de variâncias. Ciência Agrotécnica, v.28, p.381-388, 2004.

LÔBO, R.N.B.; VILLELA, L.C.V.; LÔBO, A.M.B.O.; PASSOS, J.R.S.; OLIVEIRA, A.A. Parâmetros genéticos de características estimadas da curva de crescimento de ovinos da raça Santa Inês. Revista Brasileira de Zootecnia, v.35, p.1012-1019, 2006. Supl.

MALHADO, C.H.M.; CARNEIRO, P.L.S.; SANTOS, P.F.; AZEVEDO, D.M.M.; SOUZA, J.C.; AFFONSO, P.R.M. Curva de crescimento em ovinos mestiços Santa Inês x Texel criados no Sudoeste do Estado da Bahia. Revista Brasileira de Saúde e Produção Animal [online], v.9, n.2, p.210-218, 2008.

MALHADO, C.H.M.; CARNEIRO, P.L.S.; AFFONSO, P.R.A.M.; SOUZA JÚNIOR, A.A.O.; SARMENTO, J.L.R. Growth curves in Dorper sheep crossed with the local Brazilian breeds, Morada Nova, Rabo Largo, and Santa Inês.

Small Ruminant Research, v.84, p.1621, 2009.

McMANUS, C.; EVANGELISTA, C.; FERNANDES, L.A.C.; MIRANDA, R.M.; MORENO-BERNAL, F.E.; SANTOS, N.R. Curvas de crescimento de ovinos Bergamácia criados no Distrito Federal. Revista Brasileira de Zootecnia, v.32, p.1207- 1212, 2003.

MELOUN, M.; MILITKY J. Sbírka úloh, Statistické zpracování experimentálnich dat. Pardubice: Universita Pardubice. 1996.

NOBRE, P.R.C.; ROSA, A.N; SILVA, A.N.; EVANGELISTA, S.R.M. Curvas de crescimento de gado Nelore ajustadas para diferentes frequências de pesagens. Pesquisa Agropecuária Brasileira, v.22, p. 1027-1037, 1987.

SARMENTOS, J.L.R.; REZAZZI, A.J.; SOUZA, W.H.; TORRES, R.A.; BREDA, F.C.; MENEZES, G.R.O. Estudo da curva de crescimento de ovinos Santa Inês. Revista Brasileira de Zootecnia, v.35, p.435-442, 2006. 
Rev. Bras. Saúde Prod. Anim., Salvador, v.17, n.1, p.26-36 jan./mar., 2016 http://www.rbspa.ufba.br ISSN 15199940

SAS. Statistical Analysis System user's guide. Version 9.1 ed. Cary, USA: SAS Institute, 2003.

SILVA, F.L.; ALENCAR, M.M.; FREITAS, A.R.; PACKER, I.U.; MOURÃO, G.B. Curvas de crescimento em vacas de corte de diferentes tipos biológicos. Pesquisa Agropecuária Brasileira, v.46, n.3, p.262-271, 2011.

SILVA, L.S.A.; FRAGA, A.B.; SILVA, F.L. Growth curve in Santa Inês sheep. Small Ruminant Research, v.105, p.182-185, 2012.

SILVEIRA, F.G.; SILVA, F.F.; CARNEIRO, P.L.S.; MALHADO, C.H.M; MUNIZ, J.A. Análise de agrupamento na seleção de modelos de regressão não-lineares para curvas de crescimento de ovinos cruzados. Ciência Rural, v.41, n.4, p.692-698, 2011.

SILVEIRA, F.G.; SILVA, F.F.; CARNEIRO, P.L.S.; MALHADO, C.H.M. Classificação multivariada de modelos de crescimento para grupos genéticos de ovinos de corte. Revista Brasileira de Saúde e Produção Animal [online], v.13, n1, p.62-73, 2012.

SOUZA, L.A.; CARNEIRO, P.L.S.; MALHADO, C.H.M.; PAIVA, S.R.; CAÍRES, D.N.; BARRETO, D.L.F. Curvas de crescimento em ovinos da raça morada nova criados no estado da Bahia. Revista Brasileira de Zootecnia, v.40, n.8, p.1700-1705, 2011.

SOUZA, L.D.A.; CARNEIRO, P.L.S.; MALHADO, C.H.M.; SILVA, F.F.; SILVEIRA, F.G.D. Traditional and alternative nonlinear models for estimating the growth of Morada Nova sheep. Revista Brasileira de Zootecnia, v.42, n.9, p.651-655, 2013.
TEIXEIRA NETO, M. R.; CRUZ, J. F.; CARNEIRO, P. L. S.; MALHADO, C. H. M.; BARBOSA, J. A.; SOUZA, L. E. B. Diversidade fenotípica de linhagens de ovinos Santa Inês por meio de análise multivariada. Revista Brasileira de Saúde e Produção Animal [online], v.16, n.4, p. 784-795, 2015.

TORAL, F.L.B. Número e intervalo de pesagens para estimação de parâmetros de curvas de crescimento em bovinos.

Revista Brasileira de Zootecnia, v.37, n.12, p.2120-2128, 2008.

Data de recebimento: 05/07/2015

Data de aprovação: 14/12/2015 